\title{
Magnetic and Kohn-Luttinger instabilities near a Van Hove singularity: Monolayer versus twisted bilayer graphene
}

\author{
J. González \\ Instituto de Estructura de la Materia, Consejo Superior de Investigaciones Científicas, Serrano 123, 28006 Madrid, Spain
}

(Received 28 June 2013; published 27 September 2013)

\begin{abstract}
We investigate the many-body instabilities of electrons interacting near Van Hove singularities arising in monolayer and twisted bilayer graphene. We show that a pairing instability must be dominant over the tendency to magnetic order as the Fermi level is tuned to the Van Hove singularity in the conduction band of graphene. As a result of the extended character of the saddle points in the dispersion, we find that the pairing of the electrons takes place preferentially in a channel of $f$-wave symmetry, with an order parameter vanishing at the position of the saddle points along the Fermi line. In the case of the twisted bilayers, the dispersion has instead its symmetry reduced down to the $C_{3 v}$ group and, most importantly, it leads to susceptibilities that diverge at the saddle points but are integrable along the Fermi line. This implies that a ferromagnetic instability becomes dominant in the twisted graphene bilayers near the Van Hove singularity, with a strength which is amplified as the lowest subband of the electron system becomes flatter for decreasing twist angle.
\end{abstract}

DOI: 10.1103/PhysRevB.88.125434

PACS number(s): 73.22.Pr, 74.20.Mn, 74.78.-w

\section{INTRODUCTION}

In recent years, there has been great interest in the investigation of the correlations that may arise from $e-e$ interactions in monolayer ${ }^{1-9}$ as well as in bilayer graphene. ${ }^{10-13}$ In these systems, the Coulomb repulsion between electrons constitutes the dominant interaction, placing the carbon material in a strong-coupling regime as $e^{2}$ turns out to be nominally larger than the Fermi velocity $v_{F}$ of the electrons. ${ }^{14}$ Yet the effects of electron correlations have been quite elusive, apart from the observation of the fractional quantum Hall effect in monolayer graphene ${ }^{15,16}$ and several signatures of exotic phases in bilayer graphene. ${ }^{17,18}$

The observation of superconductivity in a graphene system is a widely shared aspiration, and some proposals have been already put forward to induce a pairing instability in the carbon layer. ${ }^{19-26}$ Several experimental studies have been carried out showing the feasibility of superconducting correlations and even supercurrents in graphene contacted with superconducting electrodes. ${ }^{27,28}$ However, it is still an intriguing question whether graphene may support superconducting correlations on its own under appropriate experimental conditions.

In that respect, a suitable way of amplifying the electronic correlations may consist in tuning the Fermi level at the points with divergent density of states (so-called Van Hove singularities) that are present in the spectrum of both monolayer graphene and twisted graphene bilayers. Experimental measures of the electronic dispersion in graphene at large doping levels have shown indeed that the saddle points in the conduction band develop an extended shape that may significantly reinforce the modulation of the screened Coulomb interaction. $^{29}$ On the other hand, a Van Hove singularity has been also observed experimentally in the lowest-energy subband of the twisted bilayers, ${ }^{30,31}$ which makes them ideal systems to address the effects of the strong correlation with a minimum of electron doping.

In narrow-band electron systems, magnetic and superconducting instabilities are in general likely to appear as a consequence of the enhanced density of states near the Fermi level. The route towards superconductivity can be elaborated starting from ideas proposed long ago by Kohn and Luttinger, trying to understand whether a pairing instability could arise out of a purely repulsive interaction. ${ }^{32}$ It happens that in electron systems with anisotropic dispersion, the Coulomb interaction can be screened with different intensity along the Fermi surface, giving rise in some cases to a significant modulation of the effective interaction. Then it becomes possible that, after making the decomposition into the different modes according to the symmetry group of the Fermi surface, the couplings in some of the channels may turn out to be negative. ${ }^{33,34}$ This sign of attractive interaction is enough to trigger a superconducting instability, though the magnitude of the negative couplings may be in general so small that the critical scale for superconductivity becomes many orders of magnitude smaller than the Fermi energy.

However, in cases where the Fermi surface is close to saddle points in the electronic dispersion, the modulation displayed by the screened $e-e$ interaction can be quite strong, as a result of the divergent density of states provided by the saddle points. In models with such a Van Hove singularity in the spectrum, the critical scale for the pairing instability may be a small fraction of the typical energy scale of the band structure, leading nevertheless to much higher transition temperatures than those obtained in conventional models with electron-phonon interactions. In the context of the high- $T_{c}$ cuprate superconductors, the proximity of the Fermi level to a saddle point in the electronic dispersion ${ }^{35,36}$ has been invoked to account for several unconventional properties of the cuprates, ${ }^{37,38}$ including the $d$-wave order parameter of the superconducting condensate. ${ }^{39,40}$ Many of these features enter also in correspondence with the properties expected from microscopic theories of the cuprates, based mainly on the Hubbard model.

In the context of the honeycomb carbon lattice, an investigation of the role of the Van Hove singularities to induce a superconducting instability has been carried out in Ref. 23. The analysis was made there under the assumption of a relatively small third-neighbor hopping, still preserving an approximate 
nesting of the Fermi line passing by the saddle points. Under these conditions, the electron scattering is more intense at the momentum connecting each two inequivalent saddle points, and the superconducting instability turns out to appear in the channel with $d$-wave symmetry. A similar conclusion was reached in the analysis of Ref. 41. Anyhow, that geometry of the Fermi line does not seem to be applicable to the Van Hove singularity in the conduction band of graphene, where the extended character of the saddle points favors the scattering of electrons with vanishing momentum transfer. Then, the development of the superconducting instability takes place in general in the $f$-wave channel. ${ }^{29}$ The same symmetry of the order parameter has been also obtained in numerical studies of the Van Hove singularity under the assumption of short-range Coulomb interaction. ${ }^{42}$ In a different unrelated situation, $f$-wave symmetry has been also predicted for a superconducting instability arising in graphene at low doping levels about the charge neutrality point. ${ }^{22}$

In the present paper, we will apply the Kohn-Luttinger mechanism of superconductivity to the case where there is an extended Van Hove singularity in the electronic spectrum, as happens in the conduction band of graphene. We will provide a very general argument to show that the superconducting instability must have then $f$-wave symmetry. As the electron scattering is greatly enhanced by the extended character of the saddle points in the dispersion, the uniform magnetic susceptibility can also grow large in that situation. We will see however that the couplings measuring the effective attraction in the pairing channels are also amplified, formally diverging when the critical point for a ferromagnetic instability is approached. This explains that the pairing instability turns out to prevail over the tendency towards magnetic order in the presence of the extended Van Hove singularity.

In the case of the twisted graphene bilayers, we will see instead that they are more prone to develop a magnetic instability as they approach the regime close to the formation of flat zero-energy subbands for decreasing twist angle. ${ }^{43-45}$ We will show that the low-energy Van Hove singularity arising from the hybridization of twisted Dirac cones favors then a ferromagnetic instability in the system. This is reminiscent of the evidence of ferromagnetism found in Monte Carlo simulations of the Hubbard model in the square lattice with next-to-nearest neighbor hopping, in the limit where the Van Hove singularity undergoes a similar collapse at the bottom of the band. ${ }^{46}$ As shown below, the many-body approaches used here to describe these instabilities can be also put in correspondence with renormalization group analyses of the interacting electron system, which have also shown that ferromagnetism is a likely instability when the Fermi level is close to a Van Hove singularity, in the absence of significant nesting of the Fermi surface. ${ }^{47}$

\section{MAGNETIC AND PAIRING INSTABILITIES}

In a two-dimensional system, the Van Hove singularities in the density of states arise from the presence of saddle points in the electronic dispersion, as those shown in a typical plot for the graphene lattice in Fig. 1. For small deviation $\mathbf{k}$ of the momentum with respect to the center of each saddle point, the

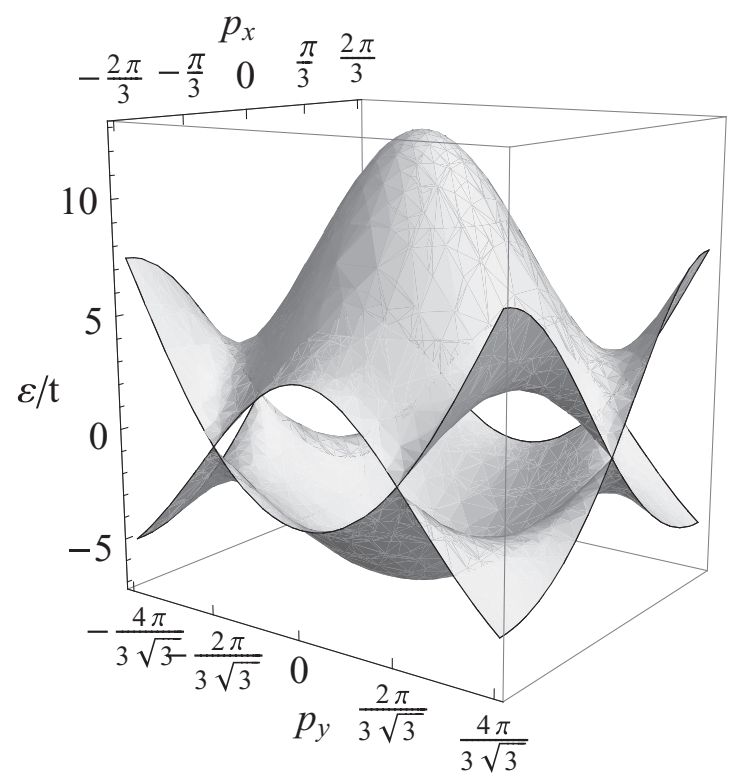

FIG. 1. Plot of the dispersion of the conduction and valence bands from a tight-binding model of graphene (energy is measured in units of the nearest-neighbor hopping $t$ and momentum in units of the inverse of the $\mathrm{C}-\mathrm{C}$ distance).

dispersion can be approximated by

$$
\varepsilon(\mathbf{k}) \approx \alpha k_{x}^{2}-\beta k_{y}^{2} \text {. }
$$

As a consequence of the flatness of the band around the saddle points, a number of susceptibilities diverge at the Van Hove singularity, with relative strengths that depend on the particular values of the $\alpha$ and $\beta$ parameters. The particlehole susceptibility $\chi_{\mathrm{ph}}(\mathbf{q}, \omega)$ at vanishing momentum transfer is given for instance by

$$
\chi_{\mathrm{ph}}(\mathbf{0}, \omega) \approx \frac{1}{4 \pi^{2}} \frac{1}{\sqrt{\alpha \beta}} \ln \left(\frac{\Lambda_{0}}{\omega+\mu}\right),
$$

where $\mu$ measures the deviation of the Fermi level with respect to the Van Hove singularity and $\Lambda_{0}$ is a high-energy cutoff. The particle-hole susceptibility at momentum transfer $\mathbf{Q}$ connecting two inequivalent saddle points also diverges as

$$
\chi_{\mathrm{ph}}(\mathbf{Q}, \omega) \approx \frac{1}{2 \sqrt{3} \pi^{2}} \frac{c^{\prime}}{\alpha+\beta} \ln \left(\frac{\Lambda_{0}}{\omega+\mu}\right)
$$

with a prefactor given in the case of $\alpha>3 \beta>0$ by

$$
c^{\prime}=\ln \left(\frac{1+\sqrt{\beta / 3 \alpha}}{1-\sqrt{\beta / 3 \alpha}}\right)+\ln \left(\frac{1+\sqrt{3 \beta / \alpha}}{1-\sqrt{3 \beta / \alpha}}\right) .
$$

The singularity in the expression (4) reflects the instance of perfect nesting of the Fermi line, which corresponds in the honeycomb lattice to $\alpha=3 \beta$ or $\alpha=\beta / 3$. Finally, the particleparticle susceptibility $\chi_{\mathrm{pp}}(\mathbf{q}, \omega)$ at vanishing total momentum $\mathbf{q}$ of the pair of electrons diverges as

$$
\chi_{\mathrm{pp}}(\mathbf{0}, \omega) \approx \frac{1}{4 \pi^{2}} \frac{1}{\sqrt{\alpha \beta}} \ln \left(\frac{\Lambda_{0}}{\omega+\mu}\right) .
$$

The large growth of the susceptibilities as $\mu \rightarrow 0$ leads to the divergence of several response functions as one approaches the Van Hove singularity. The strongest divergence 
among them dictates the character of the dominant electronic instability in the system. In this respect, there are two different scenarios, depending on the relative strength of the particlehole susceptibilities at momentum transfer $\mathbf{0}$ and $\mathbf{Q}$. When $\chi_{\mathrm{ph}}(\mathbf{Q}, \omega)>\chi_{\mathrm{ph}}(\mathbf{0}, \omega)$, the tendency towards a spin-densitywave instability at momentum $\mathbf{Q}$ prevails over a ferromagnetic instability. In that case, however, a superconducting instability may be also viable, due to the anisotropy created in the effective $e-e$ interaction by the large momentum-dependent screening near the Van Hove singularity. This is actually the possibility that was studied in Ref. 23. In those circumstances, it was shown that the dominant superconducting instability takes place in a $d$-wave channel, which corresponds to the degenerate representation $\{\cos (2 m \theta), \sin (2 m \theta)\}$ ( $m$ integer, $2 m$ not a multiple of 3 ) of the point symmetry group $C_{6 v}$.

The other scenario corresponds to the case in which $\chi_{\mathrm{ph}}(\mathbf{Q}, \omega)<\chi_{\mathrm{ph}}(\mathbf{0}, \omega)$. A ferromagnetic instability may arise then in the system, as illustrated in the analysis of the square lattice by renormalization group methods near the Van Hove singularity $^{47}$ and supported by Monte Carlo simulations. ${ }^{46}$ As mentioned before, the case of largest strength of the particle-hole susceptibility $\chi_{\mathrm{ph}}(\mathbf{0}, \omega)$ is in general the relevant instance for graphene monolayer and bilayer systems. We will see in what follows that a superconducting instability is also possible in this case, but with an order parameter which has preferentially $f$-wave symmetry.

An important point regarding the many-body theory of electrons near a Van Hove singularity is that all the momentum dependence of the interaction potential is irrelevant when scaling the theory towards the low-energy limit. This is the reflection of the intense screening from the divergent density of states, that reduces the effective interaction at low energies to a purely local component in real space. This can be seen from inspection of the effective action, written in terms of creation [annihilation] operators $\psi_{\sigma}^{\dagger}(\mathbf{k}, t)\left[\psi_{\sigma}(\mathbf{k}, t)\right]$ for electrons with $\operatorname{spin} \sigma=\uparrow, \downarrow$ as

$$
\begin{aligned}
S= & \int d t d^{2} k \sum_{\sigma}\left[\psi_{\sigma}^{\dagger}(\mathbf{k}, t) i \partial_{t} \psi_{\sigma}(\mathbf{k}, t)\right. \\
& \left.-\varepsilon(\mathbf{k}) \psi_{\sigma}^{\dagger}(\mathbf{k}, t) \psi_{\sigma}(\mathbf{k}, t)\right] \\
& -\frac{1}{2} \int d t d^{2} k \sum_{\sigma, \sigma^{\prime}} \rho_{\sigma}(\mathbf{k}, t) v_{\sigma \sigma^{\prime}}(\mathbf{k}) \rho_{\sigma^{\prime}}(-\mathbf{k}, t)
\end{aligned}
$$

with the Fourier transform $\rho_{\sigma}(\mathbf{k}, t)$ of the electron density given by

$$
\rho_{\sigma}(\mathbf{q}, t)=\int d^{2} k \psi_{\sigma}^{\dagger}(\mathbf{k}+\mathbf{q}, t) \psi_{\sigma}(\mathbf{k}, t) .
$$

Thus, one can check that the action (6) is invariant under the scale transformation

$$
t^{\prime}=\frac{1}{s} t, \quad \mathbf{k}^{\prime}=\sqrt{s} \mathbf{k}, \quad \psi^{\prime}=\frac{1}{\sqrt{s}} \psi,
$$

provided one keeps only the zeroth-order term in the expansion of $v_{\sigma \sigma^{\prime}}(\mathbf{k})$ in powers of the momentum. Higher orders in the power series of the potential would be affected by powers of $1 / \sqrt{s}$, with the result that they would be increasingly suppressed as $s \rightarrow \infty$. This is the limit in which the theory is scaled down to low energies, meaning that one can just remain with the constant term $v_{\perp} \equiv v_{\uparrow \downarrow}(\mathbf{0})$ for the sake of studying

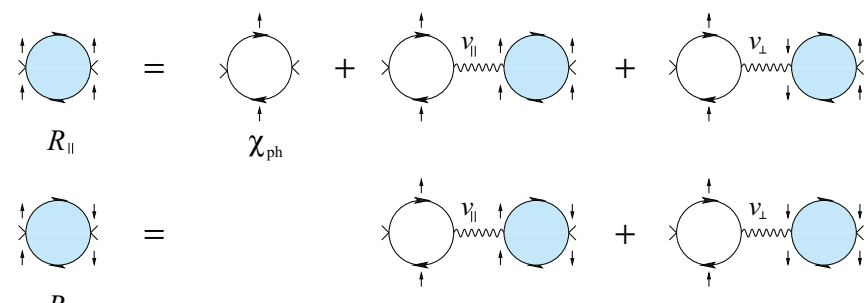

$R_{\perp}$

FIG. 2. (Color online) Self-consistent diagrammatic equations for the response functions $R_{\|}$and $R_{\perp}$ in the RPA.

the low-energy instabilities of the electron system near the Van Hove singularity.

\section{A. Ferromagnetic instability}

Focusing on the case where the largest electron-hole susceptibility is given by $\chi_{\mathrm{ph}}(\mathbf{0}, \omega)$, we can sum up in the framework of the RPA the most divergent contributions to the charge and spin response functions. These are given by correlators of the electron densities $\rho_{\uparrow}(\mathbf{q}, \omega)$ and $\rho_{\downarrow}(\mathbf{q}, \omega)$, that we define now with more generality from electron creation [annihilation] operators $\psi_{j \sigma}^{\dagger}(\mathbf{k}, \omega)\left[\psi_{j \sigma}(\mathbf{k}, \omega)\right]$ for a number of independent saddle points $j=1, \ldots N$,

$\rho_{\sigma}\left(\mathbf{q}, \omega_{q}\right)=\sum_{j, j^{\prime}} \int d^{2} k d \omega_{k} \psi_{j \sigma}^{\dagger}\left(\mathbf{k}+\mathbf{q}, \omega_{k}+\omega_{q}\right) \psi_{j^{\prime} \sigma}\left(\mathbf{k}, \omega_{k}\right)$.

The response functions for charge and spin are given respectively by

$$
\begin{aligned}
R_{c}(\mathbf{q}, \omega)= & \left\langle[ \rho _ { \uparrow } ( \mathbf { q } , \omega ) + \rho _ { \downarrow } ( \mathbf { q } , \omega ) ] \left[\rho_{\uparrow}(-\mathbf{q},-\omega)\right.\right. \\
& \left.\left.+\rho_{\downarrow}(-\mathbf{q},-\omega)\right]\right\rangle, \\
R_{S}(\mathbf{q}, \omega)= & \left\langle[ \rho _ { \uparrow } ( \mathbf { q } , \omega ) - \rho _ { \downarrow } ( \mathbf { q } , \omega ) ] \left[\rho_{\uparrow}(-\mathbf{q},-\omega)\right.\right. \\
& \left.\left.-\rho_{\downarrow}(-\mathbf{q},-\omega)\right]\right\rangle .
\end{aligned}
$$

In general, the $e-e$ interaction may be mediated by a potential $v_{\|}(\mathbf{q})$ between electrons with parallel spin and $v_{\perp}(\mathbf{q})$ for electrons with opposite spin projections. In the RPA, the response functions $R_{\|}=\left(R_{c}+R_{s}\right) / 2$ and $R_{\perp}=\left(R_{c}-\right.$ $\left.R_{S}\right) / 2$ must obey the self-consistent equations represented diagrammatically in Fig. 2. We have then

$$
\begin{gathered}
R_{\|}(\mathbf{0}, \omega)=2 N \chi_{\mathrm{ph}}(\mathbf{0}, \omega)-N \chi_{\mathrm{ph}}(\mathbf{0}, \omega) v_{\|}(\mathbf{0}) R_{\|}(\mathbf{0}, \omega) \\
-N \chi_{\mathrm{ph}}(\mathbf{0}, \omega) v_{\perp}(\mathbf{0}) R_{\perp}(\mathbf{0}, \omega), \\
R_{\perp}(\mathbf{0}, \omega)=-N \chi_{\mathrm{ph}}(\mathbf{0}, \omega) v_{\perp}(\mathbf{0}) R_{\|}(\mathbf{0}, \omega) \\
-N \chi_{\mathrm{ph}}(\mathbf{0}, \omega) v_{\|}(\mathbf{0}) R_{\perp}(\mathbf{0}, \omega) .
\end{gathered}
$$

Solving the linear system (12) and (13), we find

$$
\begin{aligned}
& R_{\|}(\mathbf{0}, \omega)=\frac{2 N \chi_{\mathrm{ph}}(\mathbf{0}, \omega)\left[1+N v_{\|}(\mathbf{0}) \chi_{\mathrm{ph}}(\mathbf{0}, \omega)\right]}{\left[1+N v_{\|}(\mathbf{0}) \chi_{\mathrm{ph}}(\mathbf{0}, \omega)\right]^{2}-\left[N v_{\perp}(\mathbf{0}) \chi_{\mathrm{ph}}(\mathbf{0}, \omega)\right]^{2}}, \\
& R_{\perp}(\mathbf{0}, \omega)=\frac{-2 N^{2} v_{\perp}(\mathbf{0})\left[\chi_{\mathrm{ph}}(\mathbf{0}, \omega)\right]^{2}}{\left[1+N v_{\|}(\mathbf{0}) \chi_{\mathrm{ph}}(\mathbf{0}, \omega)\right]^{2}-\left[N v_{\perp}(\mathbf{0}) \chi_{\mathrm{ph}}(\mathbf{0}, \omega)\right]^{2}} .
\end{aligned}
$$


We can go back now to the charge and spin response functions, obtaining the result

$$
\begin{aligned}
R_{c}(\mathbf{0}, \omega) & =\frac{2 N \chi_{\mathrm{ph}}(\mathbf{0}, \omega)}{1+N\left[v_{\|}(\mathbf{0})+v_{\perp}(\mathbf{0})\right] \chi_{\mathrm{ph}}(\mathbf{0}, \omega)}, \\
R_{s}(\mathbf{0}, \omega) & =\frac{2 N \chi_{\mathrm{ph}}(\mathbf{0}, \omega)}{1+N\left[v_{\|}(\mathbf{0})-v_{\perp}(\mathbf{0})\right] \chi_{\mathrm{ph}}(\mathbf{0}, \omega)} .
\end{aligned}
$$

We find then the origin of the spin instability when $v_{\|}(\mathbf{0})<$ $v_{\perp}(\mathbf{0})$. This is the natural situation when the screening effects are so strong that the $e-e$ interaction is reduced to a purely on-site repulsion. In the context of electrons interacting near a Van Hove singularity, we have already seen that $v_{\perp}(\mathbf{0})$ is the only component of the interaction which is not irrelevant in the low-energy limit. This explains why the tendency towards uniform spin order is a natural instability in systems with a large density of states for which the interaction can be modeled by a local Coulomb repulsion.

A remarkable feature is that the ferromagnetic instability can be always reached, no matter the strength of the interaction, provided one can place the Fermi level arbitrarily close to the Van Hove singularity. Taking then the expression of the particle-hole susceptibility in (2) and assuming a local interaction with constant potential $v_{\perp}(\mathbf{p})$, the instability is found at the critical energy

$$
\omega_{c}=\Lambda_{0} \exp \left(-\frac{4 \pi^{2} \sqrt{\alpha \beta}}{N v_{\perp}}\right) .
$$

We have anyhow to bear in mind that a very low value of $\omega_{c}$ may represent the unfeasibility to observe in practice any instability, if that energy is below the resolution with which one can approach experimentally the singularity. This also includes the possible effect of disorder, that may attenuate the divergence of the density of states below a certain energy scale as we will discuss later.

Regarding the expressions (16) and (17), we point out that their coincidence with the results obtained from a scaling analysis of the Van Hove singularity ${ }^{47}$ is reassuring. That is, the present results can be also interpreted as the lowest order of a renormalization group approach to the singularity. This level of approximation still misses relevant effects, some of them reinforcing the divergent density of states (renormalization of the saddle point dispersion) and others tending to weaken the electronic correlations (renormalization of the quasiparticle peak) ${ }^{48}$ Nevertheless, Eq. (18) can be used at least to estimate the order of magnitude of the critical energy scale. Full nonperturbative studies of the low-energy instabilities arising near a Van Hove singularity (as those carried out by means of Monte Carlo simulations in the square lattice ${ }^{46}$ ) have also certified the existence of the ferromagnetic phase, in regions of the phase diagram where the condition $\chi_{\mathrm{ph}}(\mathbf{Q}, \omega)<\chi_{\mathrm{ph}}(\mathbf{0}, \omega)$ is satisfied.

\section{B. Pairing instability}

A pairing instability is also possible when the Fermi level is close to the Van Hove singularity, as the strong screening effects make the effective $e$-e interaction quite anisotropic as a function of the momenta of the electrons. The tendency towards superconducting order requires however the development of an attractive interaction in any of the channels

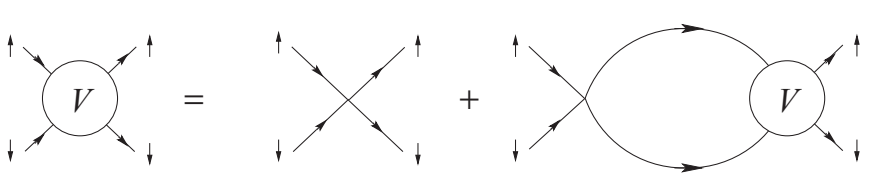

FIG. 3. Self-consistent diagrammatic equation for the BCS vertex $V$ in the ladder approximation.

for the different representations of the point symmetry group. When this happens, the pairing instability can be greatly enhanced due to the divergent density of states, already reflected in the particle-particle susceptibility given by Eq. (5).

The pairing instability can be studied by looking at the behavior of the so-called BCS vertex, that is the four-fermion interaction vertex for vanishing total momentum and spin of the incoming electrons. Such a function can be parametrized in terms of the angles $\theta$ and $\theta^{\prime}$ of the respective momenta of the spin-up incoming and outgoing electrons. Henceforth we will therefore denote the BCS vertex by $V\left(\theta, \theta^{\prime} ; \omega\right), \omega$ being the energy of the pair of electrons.

Adopting a methodology similar to that for the ferromagnetic instability, we will seek to sum up the most divergent contributions to the BCS vertex function. These can be encoded in the diagrammatic equation represented in Fig. 3, where the second term on the right-hand side accounts for the divergence of the particle-particle susceptibility. The particleparticle loop involves an integration in momentum space, that can be parametrized in terms of the differential elements $d k_{\|}$ and $d k_{\perp}$, longitudinal and normal, respectively, to the lines of constant energy. Alternatively, one can pass to integration variables defined by the energy $\varepsilon$ of the contour lines and the angle $\theta$ along them. Thus, we end up with the self-consistent equation

$$
\begin{aligned}
V\left(\theta, \theta^{\prime} ; \omega\right)= & V_{0}\left(\theta, \theta^{\prime}\right)-\frac{1}{(2 \pi)^{2}} \int_{0}^{\Lambda} d \varepsilon \int_{0}^{2 \pi} d \theta^{\prime \prime} \frac{\partial k_{\perp}}{\partial \varepsilon} \frac{\partial k_{\|}}{\partial \theta^{\prime \prime}} \\
& \times V_{0}\left(\theta, \theta^{\prime \prime}\right) \frac{1}{\varepsilon-\frac{\omega}{2}} V\left(\theta^{\prime \prime}, \theta^{\prime} ; \omega\right) .
\end{aligned}
$$

At this point, one can make contact with the more powerful scaling approach by differentiating with respect to the highenergy cutoff $\Lambda$ and applying self-consistency to the right-hand side of the equation. Then we get

$$
\begin{aligned}
& \frac{\partial V\left(\theta, \theta^{\prime} ; \omega\right)}{\partial \Lambda} \\
& =-\frac{1}{(2 \pi)^{2}} \int_{0}^{2 \pi} d \theta^{\prime \prime} \frac{\partial k_{\perp}}{\partial \varepsilon} \frac{\partial k_{\|}}{\partial \theta^{\prime \prime}} V\left(\theta, \theta^{\prime \prime} ; \omega\right) \frac{1}{\Lambda} V\left(\theta^{\prime \prime}, \theta^{\prime} ; \omega\right) .
\end{aligned}
$$

This is precisely the equation that one obtains in a renormalization group approach to the pairing instability and, in the present context, it has the advantage of allowing a proper consideration of the effect of the divergent density of states upon scaling in the low-energy limit. The density of states can be actually expressed as

$$
n(\Lambda)=\frac{1}{(2 \pi)^{2}} \int_{0}^{2 \pi} d \theta^{\prime \prime} \frac{\partial k_{\perp}}{\partial \varepsilon} \frac{\partial k_{\|}}{\partial \theta^{\prime \prime}},
$$

where the integral is carried out along a contour line of energy $\Lambda$. Then we can write Eq. (20) in simpler form by passing to 
the angular variable

$$
\phi(\theta)=\frac{1}{2 \pi n(\Lambda)} \int_{0}^{\theta} d \theta^{\prime \prime} \frac{\partial k_{\perp}}{\partial \varepsilon} \frac{\partial k_{\|}}{\partial \theta^{\prime \prime}} .
$$

In terms of the new function $\widetilde{V}\left(\phi, \phi^{\prime} ; \omega\right)=V\left(\theta, \theta^{\prime} ; \omega\right)$, the scaling equation reads

$$
\Lambda \frac{\partial \widetilde{V}\left(\phi, \phi^{\prime} ; \omega\right)}{\partial \Lambda}=-\frac{n(\Lambda)}{2 \pi} \int_{0}^{2 \pi} d \phi^{\prime \prime} \widetilde{V}\left(\phi, \phi^{\prime \prime} ; \omega\right) \widetilde{V}\left(\phi^{\prime \prime}, \phi^{\prime} ; \omega\right) .
$$

The BCS vertex is a function of the frequency $\omega$ as well as of the high-energy cutoff $\Lambda$. Under the assumption of scaling, it must be actually a function of the ratio $\omega / \Lambda$, so the low-energy limit $\omega \rightarrow 0$ can be approached by solving Eq. (23) in the limit of large $\Lambda$. In the particular case of approximately constant density of states $n$ and angle-independent vertex $\widetilde{V}(\omega)$, the solution of the equation leads to a singularity for attractive interaction $\widetilde{V}<0$, with the well-known relation between the low-energy critical scale $\omega_{c}$ and the high-energy cutoff

$$
\omega_{c} \approx \Lambda_{0} \exp \{-1 / \lambda\}, \quad \lambda \equiv n\left|\widetilde{V}\left(\Lambda_{0}\right)\right| .
$$

In the proximity of the Van Hove singularity, however, we see that a pairing instability may be enhanced by the logarithmic divergence of $n(\Lambda)$, increasing significantly the value of $\omega_{c}$.

In the case of energy-dependent density of states, we can still resort to an approximation that allows us to estimate the strength of a pairing instability by using a relation like (24), in terms of a set of constant effective couplings. For that purpose, we can introduce in Eq. (20) the change of variables

$$
\begin{aligned}
\widehat{V}\left(\theta, \theta^{\prime} ; \omega\right)= & \sqrt{\frac{1}{2 \pi} \frac{\partial k_{\perp}(\theta)}{\partial \varepsilon} \frac{\partial k_{\|}(\theta)}{\partial \theta}} \\
& \times \sqrt{\frac{1}{2 \pi} \frac{\partial k_{\perp}\left(\theta^{\prime}\right)}{\partial \varepsilon} \frac{\partial k_{\|}\left(\theta^{\prime}\right)}{\partial \theta^{\prime}}} V\left(\theta, \theta^{\prime} ; \omega\right) .
\end{aligned}
$$

In terms of the redefined vertex, the scaling equation can be then approximated by

$$
\Lambda \frac{\partial \widehat{V}\left(\theta, \theta^{\prime} ; \omega\right)}{\partial \Lambda}=-\frac{1}{2 \pi} \int_{0}^{2 \pi} d \theta^{\prime \prime} \widehat{V}\left(\theta, \theta^{\prime \prime} ; \omega\right) \widehat{V}\left(\theta^{\prime \prime}, \theta^{\prime} ; \omega\right) .
$$

The vertex (25) is actually the starting point of usual analyses of the pairing instabilities near a Van Hove singularity, ${ }^{49,50}$ where the couplings computed for the effective attraction can be interpreted in the framework of the standard BCS theory.

The integration of Eq. (26) can be facilitated by expanding the vertex $\widehat{V}\left(\theta, \theta^{\prime} ; \omega\right)$ in terms of the modes $\Psi_{m}^{(\gamma)}(\theta)$ for the different representations $\gamma$ of the point symmetry group,

$$
\widehat{V}\left(\theta, \theta^{\prime} ; \omega\right)=\sum_{\gamma, m, n} V_{m, n}^{(\gamma)} \Psi_{m}^{(\gamma)}(\theta) \Psi_{n}^{(\gamma)}\left(\theta^{\prime}\right) .
$$

We arrive then at a set of equations for each representation $\gamma$ :

$$
\Lambda \frac{\partial V_{m, n}^{(\gamma)}}{\partial \Lambda}=-\sum_{s} V_{m, s}^{(\gamma)} V_{s, n}^{(\gamma)}
$$

It becomes clear that, for positive initial values of $V_{m, n}^{(\gamma)}$, the couplings fade away in the low-energy regime approached as $\Lambda \rightarrow \infty$. In the present case of highly anisotropic screening, some of the channels may start however with an attractive effective interaction. Then, as observed from Eq. (28), this will be enough to trigger a pairing instability at a low-energy critical scale like that in Eq. (24).

A sensible way of computing initial values for $V_{m, n}^{(\gamma)}$ is to start with a dressed vertex accounting for the effects of the electronhole polarization not included in the sum of Fig. 3. One can indeed perform the sum of RPA and ladder contributions obtained from iteration of the electron-hole scattering, as proposed in Ref. 49. Assuming as in the previous section a local interaction with constant potential $v_{\perp}$ in momentum space, the initial value of the BCS vertex is given in this approximation by $^{49}$

$$
\begin{aligned}
\widehat{V}_{0}\left(\theta, \theta^{\prime}\right)= & F(\theta) F\left(\theta^{\prime}\right)\left(v_{\perp}+\frac{v_{\perp}^{2} \chi_{\mathrm{ph}}\left(\mathbf{k}+\mathbf{k}^{\prime}\right)}{1-v_{\perp} \chi_{\mathrm{ph}}\left(\mathbf{k}+\mathbf{k}^{\prime}\right)}\right. \\
& \left.+\frac{v_{\perp}^{3} \chi_{\mathrm{ph}}^{2}\left(\mathbf{k}-\mathbf{k}^{\prime}\right)}{1-v_{\perp}^{2} \chi_{\mathrm{ph}}^{2}\left(\mathbf{k}-\mathbf{k}^{\prime}\right)}\right)
\end{aligned}
$$

with

$$
F(\theta)=\sqrt{\frac{1}{2 \pi} \frac{\partial k_{\perp}(\theta)}{\partial \varepsilon} \frac{\partial k_{\|}(\theta)}{\partial \theta}}
$$

and $\mathbf{k}, \mathbf{k}^{\prime}$ being the respective momenta at angles $\theta, \theta^{\prime}$ over the energy contour line. The singularity in the fractions of Eq. (29) corresponds at vanishing momentum to the ferromagnetic instability discussed in the previous section. As already mentioned, the mode expansion of (29) may lead however to a negative coupling in some of the channels, making the pairing instability to prevail at a higher critical scale, as we will see in the next section.

\section{VAN HOVE SINGULARITIES IN MONOLAYER AND TWISTED BILAYER GRAPHENE}

In all the relevant instances where a Van Hove singularity arises in graphene-based systems, the electron-hole susceptibility appears to reach its maximum peak at vanishing momentum transfer. This narrows down the possible electronic instabilities to either a tendency towards ferromagnetism or towards Cooper pairing with unconventional (preferentially $f$-wave) order parameter. The case of monolayer graphene with large electron doping is an example of the latter instance, as a result of the strong modulation of the interaction around the extended saddle points in the electronic dispersion. The tendency to magnetic order turns out to be dominant instead in the twisted graphene bilayers, given the lower degree of symmetry but larger number of saddle points in the dispersion.

\section{A. Monolayer graphene}

The electronic dispersion near the Van Hove singularity in the conduction band of graphene has been mapped in the ARPES experiments reported in Ref. 29. The saddle point dispersion around the $\mathrm{M}$ point of the Brillouin zone shows an extended character that cannot be accounted for by conventional approaches such as the LDA or $G W$ approximations, reflecting that it arises as an effect of strong electronic correlation. One can anyhow fit empirically the electronic dispersion by writing down a tight-binding Hamiltonian for electron creation (annihilation) operators $c_{i}^{\dagger}\left(c_{i}\right)$ in the 


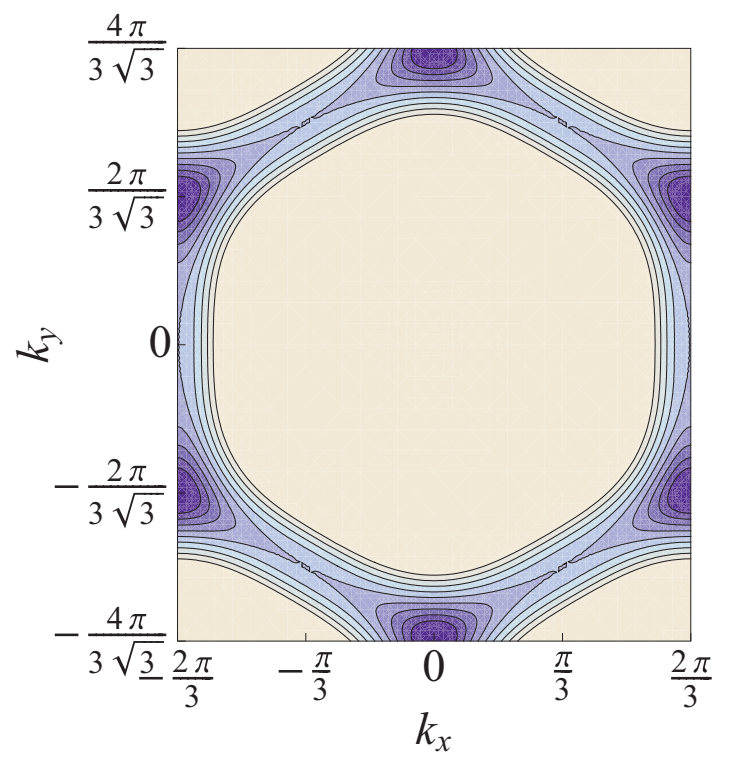

FIG. 4. (Color online) Plot of energy contour lines around the saddle points in the conduction band of graphene, obtained from a tight-binding model with first-, second-, and third-neighbor hopping parameters (momenta $k_{x}$ and $k_{y}$ are measured in units of the inverse of the $\mathrm{C}-\mathrm{C}$ distance).

honeycomb lattice

$$
H=-t \sum_{i, j} c_{i}^{\dagger} c_{j}-t^{\prime} \sum_{i, j} c_{i}^{\dagger} c_{j}-t^{\prime \prime} \sum_{i, j} c_{i}^{\dagger} c_{j},
$$

where the sums account respectively for hopping between first-, second-, and third-neighbor carbon atoms. The hopping parameters giving the best fit to the experimental results can be found in Ref. 29. A plot of the energy contour lines obtained from the diagonalization of (31) near the Van Hove singularity in the conduction band is shown in Fig. 4.

The extended character of the saddle points seen in Fig. 4 leads to a marked enhancement of the electron-hole susceptibility at vanishing momentum transfer. Moreover, the BCS vertex develops a strong modulation along the Fermi line passing near the saddle points, as observed in Fig. 5. The oscillations are translated to the initial condition (29) for the vertex, in such a way that the periodic behavior along the Fermi line is dominated by the first harmonics:

$$
\widehat{V}_{0}(\theta, \theta)=c_{0}+c_{2} \cos (2 \theta)+c_{4} \cos (4 \theta)+c_{6} \cos (6 \theta)+\cdots,
$$
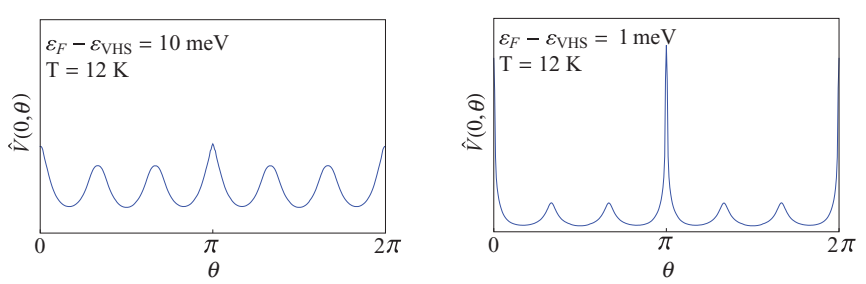

FIG. 5. (Color online) Modulation of the BCS vertex as the angle $\theta$ makes a complete turn along the Fermi line, for different shifts of the Fermi energy $\varepsilon_{F}$ with respect to the level of the Van Hove singularity $\varepsilon_{\mathrm{VHS}}$ and temperature $T=12 \mathrm{~K}$.

$$
\widehat{V}_{0}(0, \theta)=c_{0}^{\prime}+c_{2}^{\prime} \cos (2 \theta)+c_{4}^{\prime} \cos (4 \theta)+c_{6}^{\prime} \cos (6 \theta)+\cdots,
$$

$$
\begin{aligned}
\widehat{V}_{0}(\theta,-\theta)= & c_{0}^{\prime \prime}+c_{2}^{\prime \prime} \cos (2 \theta)+c_{4}^{\prime \prime} \cos (4 \theta) \\
& +c_{6}^{\prime \prime} \cos (6 \theta)+\cdots .
\end{aligned}
$$

The expansions (32)-(34) match well with the periodicity of the modes for the irreducible representations of the $C_{6 v}$ symmetry group. Four of them are one-dimensional, with respective sets of basis functions given by $\{\cos (6 n \theta)\}$, $\{\sin (6 n \theta)\},\{\cos [(6 n+3) \theta]\}$, and $\{\sin [(6 n+3) \theta]\}$ (n being always an integer). The remaining two representations are twodimensional, corresponding to the sets $\{\cos (m \theta), \sin (m \theta)\}$, with the integer $m$ running over all values that are not multiples of 3 and which are odd for one of the representations and even for the other. The dominant terms in (32)-(34) can be accounted for by approximating the BCS vertex with the first modes of the irreducible representations:

$$
\begin{aligned}
\widehat{V}(\theta, & \left.\theta^{\prime} ; \omega\right) \\
= & V_{0,0}+2 V_{2,2}\left[\cos (2 \theta) \cos \left(2 \theta^{\prime}\right)+\sin (2 \theta) \sin \left(2 \theta^{\prime}\right)\right] \\
& +2 V_{2,4}\left[\cos (2 \theta) \cos \left(4 \theta^{\prime}\right)-\sin (2 \theta) \sin \left(4 \theta^{\prime}\right)+\theta \leftrightarrow \theta^{\prime}\right] \\
& +2 V_{3,3} \sin (3 \theta) \sin \left(3 \theta^{\prime}\right)+2 V_{3,3}^{\prime} \cos (3 \theta) \cos \left(3 \theta^{\prime}\right) \\
& +\sqrt{2} V_{0,6}\left[\cos (6 \theta)+\cos \left(6 \theta^{\prime}\right)\right]+\cdots .
\end{aligned}
$$

Comparing (35) with (32)-(34), one can draw easily the correspondence $c_{6}=4 V_{2,4}-V_{3,3}+2 \sqrt{2} V_{0,6}, c_{2}^{\prime}=$ $2 V_{2,2}+2 V_{2,4}, c_{4}^{\prime}=2 V_{2,4}, c_{6}^{\prime}=\sqrt{2} V_{0,6}$, and $c_{2}^{\prime \prime}=4 V_{2,4}, c_{4}^{\prime \prime}=$ $2 V_{2,2}, c_{6}^{\prime \prime}=V_{3,3}+2 \sqrt{2} V_{0,6}$.

Using very simple arguments, it is possible to show that at least one of the couplings in the expansion (35) must be negative, for the particular case of dispersion with the shape depicted in Fig. 4. We may take for instance two specific pairs of angles for which the BCS vertex is amplified by electronhole scattering, namely

$$
\widehat{V}\left(\frac{\pi}{6}, \frac{\pi}{6} ; \omega\right) \approx V_{0,0}+2 V_{2,2}-4 V_{2,4}+2 V_{3,3}-2 \sqrt{2} V_{0,6}
$$

and

$$
\widehat{V}\left(\frac{\pi}{2},-\frac{\pi}{2} ; \omega\right) \approx V_{0,0}+2 V_{2,2}-4 V_{2,4}-2 V_{3,3}-2 \sqrt{2} V_{0,6} .
$$

Looking now at the initial condition given by Eq. (29), it becomes clear that

$$
\widehat{V}_{0}\left(\frac{\pi}{6}, \frac{\pi}{6}\right)<\widehat{V}_{0}\left(\frac{\pi}{2},-\frac{\pi}{2}\right),
$$

since $\widehat{V}_{0}(\pi / 2,-\pi / 2)$ is enhanced by the term depending on $\chi_{\mathrm{ph}}\left(\mathbf{k}+\mathbf{k}^{\prime}\right)$ in $(29)$ at $\mathbf{k}^{\prime}=-\mathbf{k}$, while $\widehat{V}_{0}(\pi / 6, \pi / 6)$ is enhanced by the weaker term that depends on $\chi_{\mathrm{ph}}\left(\mathbf{k}-\mathbf{k}^{\prime}\right)$ at $\mathbf{k}^{\prime}=\mathbf{k}$. On the other hand, we have

$$
\widehat{V}\left(\frac{\pi}{6}, \frac{\pi}{6} ; \omega\right)-\widehat{V}\left(\frac{\pi}{2},-\frac{\pi}{2} ; \omega\right) \approx 4 V_{3,3},
$$

implying that $V_{3,3}$ must be negative.

It can be checked that $V_{3,3}$ is actually the dominant negative coupling among the terms that appear in the expression (35). We arrive therefore at the conclusion that the $f$ wave must be the symmetry of the dominant pairing instability at weak coupling, when only the first terms are significant in the 


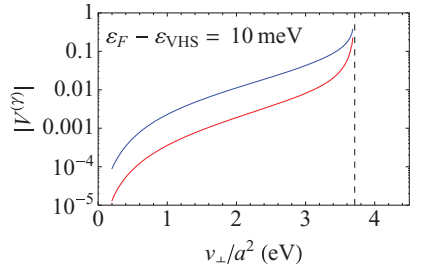

(a)

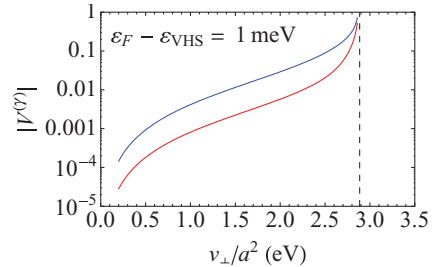

(b)
FIG. 6. (Color online) Plot of the absolute value of the negative couplings $V^{(\gamma)}$ in the channels with $\sin (3 \theta)$ symmetry (upper curves) and $\cos (3 \theta)$ symmetry (lower curves) as functions of the ratio of the potential $v_{\perp}$ to the square of the $\mathrm{C}-\mathrm{C}$ distance $a$. The dashed lines represent the location of the singularities in the fractions of the expression (29).

expansion of the vertex. This is in agreement with the results reported in Ref. 29 near the Van Hove singularity in the conduction band of graphene, where was found a dominant superconducting instability with the $f$-wave order parameter over most of the phase diagram [we note that the instability was assigned in that reference to the representation with $\cos (3 \theta)$ symmetry, consequent with the fact that the axes were rotated by $\pi / 2$ with respect to the present notation]. The actual values of the negative couplings can be seen for two different doping levels in Fig. 6, which represents the projections $V^{(\gamma)}$ of the vertex (29) after its numerical evaluation in a grid of $800 \times 800$ points in the Brillouin zone of the honeycomb lattice.

Very close to the Van Hove singularity, there could be still room for a different pairing instability with $d$-wave symmetry, ${ }^{29}$ though the analysis cannot be based then on a simple picture such as that from expression (35). Remaining otherwise with the weak-coupling expansion, the scaling equation (28) becomes in the sector of the representation $\{\cos (m \theta), \sin (m \theta)\}$ for $m$ even

$$
\Lambda \frac{\partial}{\partial \Lambda}\left(\begin{array}{ll}
V_{2,2} & V_{2,4} \\
V_{4,2} & V_{4,4}
\end{array}\right) \approx-\left(\begin{array}{ll}
V_{2,2} & V_{2,4} \\
V_{4,2} & V_{4,4}
\end{array}\right)\left(\begin{array}{ll}
V_{2,2} & V_{2,4} \\
V_{4,2} & V_{4,4}
\end{array}\right)
$$

This equation can be integrated by passing to the eigenvalues of the matrix of couplings,

$$
\lambda_{1,2}=\frac{1}{2}\left(V_{2,2}+V_{4,4}\right) \pm \frac{1}{2} \sqrt{\left(V_{2,2}-V_{4,4}\right)^{2}+4 V_{2,4}^{2}} .
$$

In the present situation where the electron-hole polarization is largest at vanishing momentum transfer, both couplings $V_{2,2}$ and $V_{4,4}$ are positive and comparable in the expansion (35). This means that both eigenvalues turn out to be positive, so that a $d$-wave instability cannot exist over most of the phase diagram, away from the strong-coupling regime in which $v_{\perp} \chi_{\mathrm{ph}}$ is close to the poles in the expression (29). The same consideration can be applied to a $p$-wave instability. It is only for doping levels very close to the Van Hove singularity, where the simple approximation (35) does not apply and many more modes start to contribute significantly to the expansion, that other instabilities apart from the mentioned $f$ wave may come into play. The precise analysis depends then on the particular shape of the dispersion but, as the numerical computation of Ref. 29 shows, the instability with $f$-wave symmetry appears to be dominant even for relatively low values of the doping level $\mu$, until the Fermi energy is tuned within $\sim 0.1 \mathrm{meV}$ about the Van Hove singularity.

At this point, it is interesting to compare the above results with those obtained in Ref. 23 for the case of largest electronhole scattering at a momentum $\mathbf{Q}$ connecting the saddle points in the electron dispersion. Under the assumption that $\chi_{\mathrm{ph}}(\mathbf{Q}, \omega)>\chi_{\mathrm{ph}}(\mathbf{0}, \omega)$, it was shown there that the couplings $V_{2,2}$ and $V_{2,4}$ are both negative, implying the development of a dominant pairing instability always with $d$-wave symmetry. The prevalence of $\chi_{\mathrm{ph}}(\mathbf{Q}, \omega)$ requires however a condition of approximate nesting of the Fermi line near the saddle points. As we have seen, this is very far from being realized in the conduction band of graphene, which stresses the role of a largest electron-hole scattering at $\mathbf{q}=0$ to account for the pairing instabilities found in the present case.

In principle, a ferromagnetic instability could also compete with the tendency to Cooper pairing in the presence of a large susceptibility $\chi_{\mathrm{ph}}(\mathbf{0}, \omega)$. At a given temperature and doping level, there is a critical interaction $\left(v_{\perp}\right)_{c}$ at which the ferromagnetic instability can take place, determined by the location of the singularity in the fractions of the expression (29). It is important to note however that the negative couplings $V^{(\gamma)}$ derived for the pairing instability diverge as that critical interaction strength is approached. This is clearly appreciated in the evolution of the couplings shown in Fig. 6. That is, the extended character of the saddle points makes the singularity in the vertex (29) nonintegrable along the Fermi line. This means that, before the temperature $T_{\text {ferro }}$ of the singularity for a given critical $\left(v_{\perp}\right)_{c}$ is reached, the critical temperature $T_{c}$ for the pairing instability is able to exceed that value of $T_{\text {ferro }}$ as the coupling $V^{(\gamma)}$ diverges [in the limit $v_{\perp} \rightarrow\left(v_{\perp}\right)_{c}$ ] in the expression

$$
k_{B} T_{c} \approx \Lambda_{0} \exp \left\{-1 /\left|V^{(\gamma)}\right|\right\} .
$$

Consequently, it is clear that the pairing instability must prevail over the ferromagnetic instability, in this particular case of the Van Hove singularity in the conduction band of graphene.

\section{B. Twisted graphene bilayer}

Band structure. Twisted graphene bilayers are a class of coupled graphene layers in which there is a relative rotation between the symmetry axes of the two carbon sheets. This gives rise to characteristic moiré patterns showing the periodic repetition of an alternating stacking with the form of a hexagonal superlattice, ${ }^{43}$ as observed in some experimental samples of graphene bilayers. ${ }^{51,52}$ We are going to deal here with a description of the twisted bilayers for relatively large period of the moiré pattern, which will allow us to apply a continuum approach to the interactions among the large number of atoms in the unit cell of the superlattice. This has in general primitive vectors $\mathbf{L}_{ \pm}=L(\sqrt{3} / 2, \pm 1 / 2)$, where the period $L$ is given in terms of the twist angle $\theta$ by the relation $L=a_{0} /[2 \sin (\theta / 2)]\left(a_{0}\right.$ being the lattice constant of graphene). Starting from perfect Bernal stacking at $\theta=\pi / 3$, the values of the twist angle consistent with a commensurate superlattice are quantized in terms of a pair of integers $(n, m),{ }^{53,54}$ being constrained by the condition

$$
\cos (\theta)=\frac{1}{2} \frac{n^{2}+m^{2}+4 m n}{n^{2}+m^{2}+m n} .
$$




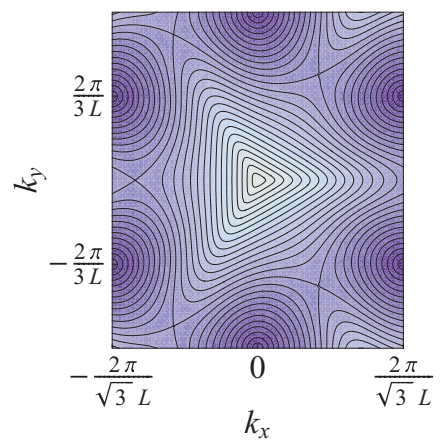

(a)

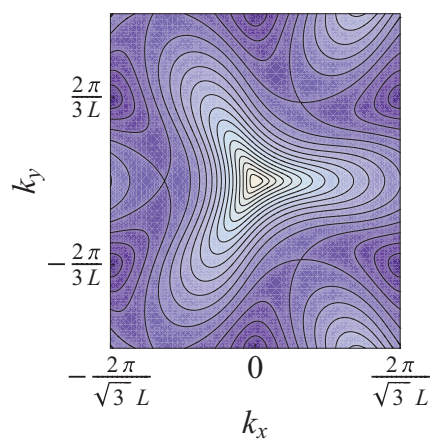

(b)

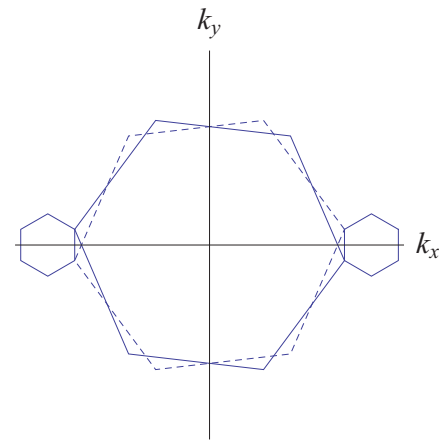

(c)

FIG. 7. (Color online) (a) and (b): Plot of energy contour lines showing the saddle points in the lowest-energy subband of twisted graphene bilayers corresponding respectively to $n=10\left(\theta \approx 3.15^{\circ}\right)$ and $n=22\left(\theta \approx 1.47^{\circ}\right)$ in the sequence (44). (c) Scheme showing the relative rotation of the Brillouin zones of the layers (large hexagons) and the relative position of the two regions (small hexagons) making up the Brillouin zone of the twisted bilayer.

In what follows, we will focus on the limit of small twist angle, which can be approached by taking $m=n+1$ and increasing $n$. This choice gives rise to the sequence of periods

$$
L_{n}=\sqrt{3 n^{2}+3 n+1} a_{0}
$$

for integer $n \geqslant 0 .{ }^{43}$

The band structure of the twisted bilayer can be obtained by realizing first that there must be a relative twist between the Brillouin zones of the two carbon layers, given by the angle of rotation $\theta$. This leads to a mismatch in the position of the respective $K$ points which, if originally placed at $\mathbf{K}=\left(4 \pi / 3 a_{0}, 0\right)$, can be taken as shifted in opposite directions by $\pm \Delta \mathbf{K} / 2=(0, \pm|\mathbf{K}| \sin (\theta / 2))$ [see Fig. 7(c)]. Next, one has to account for the hybridization of states in the $\pi$ bands of the two layers, which can undergo scattering with a momentum transfer dictated by the periodic structure of the moiré superlattice. This leads to folding of the bands of the twisted bilayer into an hexagonal Brillouin zone, with reciprocal vectors $\mathbf{Q}_{1,2}=(2 \pi / L)( \pm 1 / \sqrt{3}, 1){ }^{43}$

For relatively large values of $L$ compared to $a_{0}$, the low-energy physics of the twisted bilayer can be extracted from the hybridization between states around the respective Dirac points of the two layers rotated by a small twist $\theta$. In this approach, one can make a microscopic average of the tunneling amplitude between the two graphene layers, represented by smooth interlayer potentials $V_{A A^{\prime}}(\mathbf{r}), V_{A B^{\prime}}(\mathbf{r})$, and $V_{B A^{\prime}}(\mathbf{r})$ accounting for the modulated hopping between sublattices $A, B$ of one layer and $A^{\prime}, B^{\prime}$ of the other layer. In the space of four-component spinors $\left(\psi_{A}, \psi_{B}, \psi_{A^{\prime}}, \psi_{B^{\prime}}\right)$ made of the electronic amplitudes on each sublattice of the two graphene layers, the Hamiltonian can be written in the form ${ }^{45}$

$$
H=v_{F}\left(\begin{array}{cccc}
0 & -i \partial_{x}-\partial_{y}+i \Delta|\mathbf{K}| / 2 & V_{A A^{\prime}}(\mathbf{r}) & V_{A B^{\prime}}(\mathbf{r}) \\
-i \partial_{x}+\partial_{y}-i \Delta|\mathbf{K}| / 2 & 0 & V_{B A^{\prime}}(\mathbf{r}) & V_{A A^{\prime}}(\mathbf{r}) \\
V_{A A^{\prime}}^{\star}(\mathbf{r}) & V_{B A^{\prime}}^{\star}(\mathbf{r}) & 0 & -i \partial_{x}-\partial_{y}-i \Delta|\mathbf{K}| / 2 \\
V_{A B^{\prime}}^{\star}(\mathbf{r}) & V_{A A^{\prime}}^{\star}(\mathbf{r}) & -i \partial_{x}+\partial_{y}+i \Delta|\mathbf{K}| / 2 & 0
\end{array}\right)
$$

This provides the pertinent construction around a pair of Dirac points shifted by $\pm \Delta|\mathbf{K}| / 2$ with respect to the original $K$ points of the graphene layers. One has anyhow to bear in mind that the complete spectrum must be obtained by adding the contribution of a similar Hamiltonian representing the hybridization of the Dirac cones at the opposite $K$ points, which can be obtained by reversing the sign of the $x$ variable and exchanging the two layers in Eq. (45).

The lowest-energy subbands of the twisted graphene bilayers can be found by diagonalizing the Hamiltonian with a sensible representation of the interlayer potentials, in accordance with the symmetry of the hexagonal superlattice. This implies in particular the periodicity $V_{A A^{\prime}}(\mathbf{r})=$ $V_{A A^{\prime}}\left(\mathbf{r}+\mathbf{L}_{+}\right)=V_{A A^{\prime}}\left(\mathbf{r}+\mathbf{L}_{-}\right)$, and the relations $V_{A B^{\prime}}(\mathbf{r})=$
$V_{A A^{\prime}}\left(\mathbf{r}+\left(\mathbf{L}_{+}+\mathbf{L}_{-}\right) / 3\right), \quad V_{B A^{\prime}}(\mathbf{r})=V_{A A^{\prime}}\left(\mathbf{r}-\left(\mathbf{L}_{+}+\mathbf{L}_{-}\right) / 3\right)$. A common procedure is to assume that the interlayer hopping is dominated by processes with momentum transfer $\mathbf{Q}_{0}=0$ or equal to the reciprocal vectors $\mathbf{Q}_{1,2}$, so that $V_{A A^{\prime}}(\mathbf{r}) \approx$ $\left(w / v_{F}\right) \sum_{j} \exp \left(i \mathbf{Q}_{j} \cdot \mathbf{r}\right) .{ }^{43,44}$ For the lowest subband obtained using this approximation, the energy contour lines corresponding to the bilayers with $n=10$ and 22 in the sequence (44) are represented in Figs. 7(a) and 7(b). In the plots, the development of saddle points in the dispersion between each pair of neighboring Dirac points is manifest, although with a little lateral displacement that breaks the symmetry down to $C_{3 v}$.

Pairing instabilities. When analyzing the possible pairing instabilities in this system, one has to pay attention to the 
fact that the Fermi line of the twisted bilayer is made of two disconnected sections, which are related by the inversion $\mathbf{k} \rightarrow-\mathbf{k}$ as represented in Fig. 7(c). This means that we can distinguish between two different processes in the scattering of the Cooper pairs, depending on whether there is exchange or not of the two electrons from one section of the Fermi line to the other. The strength of the Coulomb interaction is different in the two cases, as the exchange of the electrons in the Cooper pair implies a large momentum transfer of the order of $|\mathbf{K}|$, while that must be of order $\sim 1 / L$ when each electron remains in the same section of the Fermi line. In the first instance, the Coulomb repulsion is given by the unscreened potential $v_{0}(\mathbf{K})=2 \pi e^{2} /|\mathbf{K}|$. In the other case, the interaction is already screened for momenta $\sim 1 / L$. This effect can be estimated from the charge density required to place the Fermi level close to the Van Hove singularity, located at an energy $\varepsilon_{\mathrm{VHS}} \sim v_{F} / L$ from the Dirac point. Applying the charge polarization $\chi_{D}(\mathbf{k})$ in the Dirac theory for a number of $N_{D}=8$ doped Dirac cones (including spin) in the twisted bilayer

$$
\chi_{D}\left(\frac{1}{L}\right) \approx \frac{N_{D} \varepsilon_{\mathrm{VHS}}}{2 \pi v_{F}^{2}} \sim \frac{N_{D}}{2 \pi v_{F} L},
$$

we can estimate the strength of the screened Coulomb potential as

$$
\begin{aligned}
v\left(\frac{1}{L}\right) & =\frac{v_{0}\left(\frac{1}{L}\right)}{1+v_{0}\left(\frac{1}{L}\right) \chi_{D}\left(\frac{1}{L}\right)} \\
& \sim \frac{2 \pi v_{F} L}{N_{D}} .
\end{aligned}
$$

The important point is that, already for a period $L_{n}$ with $n \approx 10$, the strength of the effective interaction $v\left(1 / L_{n}\right)$ is about one order of magnitude above that of the unscreened potential $v_{0}(\mathbf{K})$. Then, it is safe to neglect the influence of the scattering of Cooper pairs with large momentum transfer in the evaluation of the initial condition (29). Moreover, with the system placed in close proximity to the Van Hove singularity, the electron-hole susceptibility only experiences a significant enhancement at small momentum. Therefore, in order to study the effect of the singularities on the right-hand side of Eq. (29), it is justified to discard the second term in favor of the third, which is the only source of a possible pole.

We observe that the previous argument leading to a negative coupling $V_{3,3}$ from the relation (39) does not apply in the case of the twisted graphene bilayers, as it was based on the dominance of the term depending on $\chi_{\mathrm{ph}}\left(\mathbf{k}+\mathbf{k}^{\prime}\right)$ in Eq. (29) for monolayer graphene. In the present case, one can still decompose the BCS vertex as a series of the basis functions for irreducible representations of the $C_{3 v}$ point symmetry group. Two of these are one-dimensional, corresponding to the sets $\{\cos [(3 n) \theta]\}$ and $\{\sin [(3 n) \theta]\}$ for integer $n$, and the remaining is two-dimensional, represented by the set $\{\cos (m \theta), \sin (m \theta)\}$ for integer $m$ not being a multiple of 3 . It has to be remarked, however, that the electron-hole susceptibility lacks in the twisted bilayers the very large enhancement at vanishing momentum that was the consequence of the extended character of the Van Hove singularity in monolayer graphene. This does not prevent that some negative coupling may arise in any of the channels for the above representations, but one can anticipate

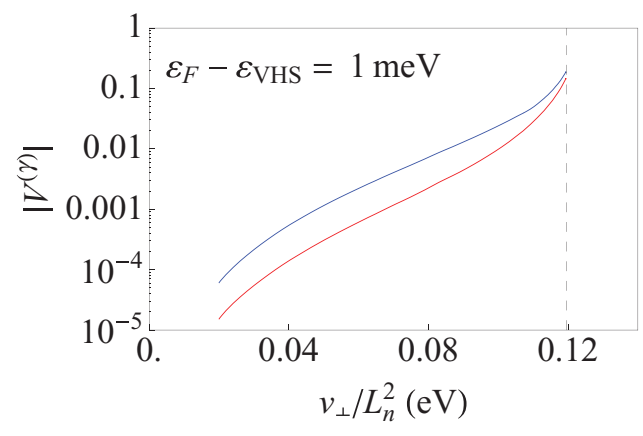

FIG. 8. (Color online) Plot of the absolute value of the negative couplings $V^{(\gamma)}$ for the irreducible representations $\{\sin [(3 n) \theta]\}$ (upper curve) and $\{\cos [(3 n) \theta]\}$ (lower curve) of the $C_{3 v}$ group, as functions of the ratio of the potential $v_{\perp}$ to the square of the lattice constant $L_{n}$ (for a twisted bilayer with $n=10$ ). The dashed line represents the point of the singularity in the spin response function for the same value of $L_{n}$.

that the effect will not correspond now to the dominance of a particular harmonic from the set of basis functions.

To estimate the scale of a possible pairing instability, we have computed the polarization $\chi_{\mathrm{ph}}(\mathbf{q})$ in a grid with $600 \times 600$ points in each hexagon of the the Brillouin zone, with the Fermi line close to the saddle points of the dispersions represented in Fig. 7. A characteristic plot of the couplings in the channels with effective attractive interaction is shown in Fig. 8. We observe that the magnitude of the couplings is significantly smaller than that found for the Van Hove singularity in the conduction band of graphene. Taking into account the correspondence (42) with the critical scale of a possible pairing instability, it is clear that the present effective attraction is too small to give rise to any observable effect in the twisted bilayers. More importantly, the couplings do not grow large when approaching the singularity in the second fraction of Eq. (29), which means that the corresponding divergence becomes integrable when computing the coefficients in the expansion (27). This implies that the tendency towards pairing cannot compete in this case with the instability in the spin response function, no matter how closely the Van Hove singularity is approached.

Ferromagnetic instability. In order to assess the strength of the magnetic instability in the system, we have evaluated $\chi_{\mathrm{ph}}(\mathbf{q})$ along high-symmetry directions down to vanishing momentum, using this time a grid with $900 \times 900$ points covering one of the hexagons of the Brillouin zone. Computing at decreasing temperatures and doping levels progressively close to the Van Hove singularity, we observe the development of a pronounced peak in the susceptibility at zero momentum, as shown in Figs. 9(a) and 10(a). This is consistent with the logarithmic divergence anticipated in the continuum approach of Sec. II. As seen in that section, such a behavior implies that, ideally, the singularity in the response function $R_{s}(\mathbf{0}, \omega)$ can be reached for any strength of the local Coulomb repulsion, simply by lowering the temperature and approaching sufficiently close the Van Hove singularity.

We remark that, in the model of the bilayer superlattice, the spin-dependent interaction $v_{\perp}$ arises at a microscopic level from the on-site Coulomb repulsion $U$ at each carbon atom. 


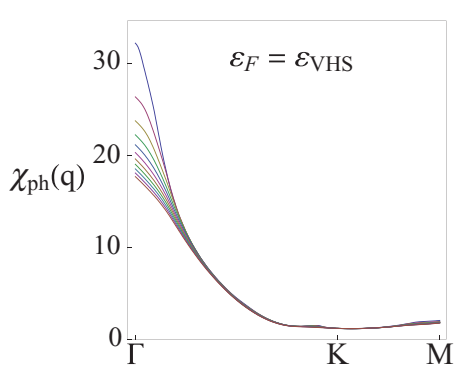

(a)

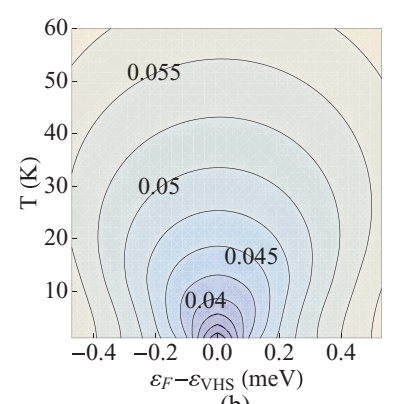

(b)
FIG. 9. (Color online) (a) Plot of the electron-hole susceptibility (in units of the inverse of eV times $L_{n}^{2}$ ) for temperatures corresponding (from top to bottom) to $k_{B} T=0.1,0.5,1,1.5,2,2.5,3,3.5,4$, $4.5,5 \mathrm{meV}$, and (b) contour lines of the critical coupling for the interaction strength $v_{\perp} / L_{n}^{2}$ (in $\mathrm{eV}$ ) as a function of temperature and doping with respect to the Van Hove singularity, for a twisted bilayer with $n=10\left(\theta \approx 3.15^{\circ}\right)$ in the sequence (44).

The eigenstates in each subband of the twisted bilayer can be written as linear combinations of the atomic orbitals in the superlattice unit cell, normalized according to the number $M$ of atoms it contains. This means that, after projecting into the lowest-energy subband, $v_{\perp} / L_{n}^{2}$ must be of the order of $U / M$, having a magnitude that decreases in the sequence of twisted bilayers as the inverse of $L_{n}^{2}$. That has to be confronted with the critical values that are predicted from the position of the pole in the response function $R_{S}(\mathbf{0}, \omega)$, which we have represented for a twisted bilayer with $n=10$ in Fig. 9(b). Assuming a magnitude of the on-site Coulomb repulsion $U \sim 10 \mathrm{eV},{ }^{55}$ we obtain $U / M \sim 0.03 \mathrm{eV}$ for that particular superlattice. We see that such an interaction strength cannot match the lowest critical couplings represented in the figure, staying in close proximity to the Van Hove singularity. Thus, for the corresponding twisted bilayer, a signature of the magnetic instability should be only expected at a temperature $\lesssim 1 \mathrm{~K}$.

We have anyhow to bear in mind that the band structure of the twisted bilayers undergoes important changes as the period $L_{n}$ increases, with a progressive reduction in the width of the lowest-energy subband. It has been actually found that such a bandwidth has a recurrent behavior, narrowing

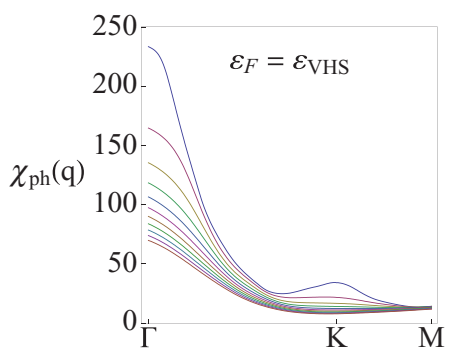

(a)

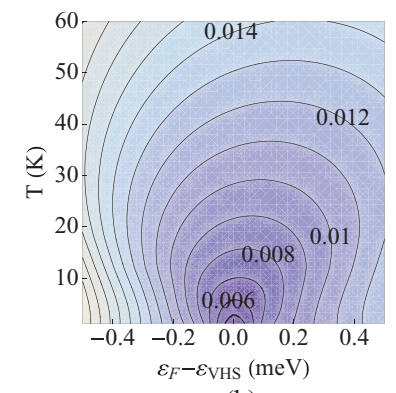

(b)
FIG. 10. (Color online) (a) Plot of the electron-hole susceptibility (in units of the inverse of eV times $L_{n}^{2}$ ) for temperatures corresponding (from top to bottom) to $k_{B} T=0.1,0.5,1,1.5,2,2.5,3,3.5,4$, $4.5,5 \mathrm{meV}$, and (b) contour lines of the critical coupling for the interaction strength $v_{\perp} / L_{n}^{2}$ (in $\mathrm{eV}$ ) as a function of temperature and doping with respect to the Van Hove singularity, for a twisted bilayer with $n=22\left(\theta \approx 1.47^{\circ}\right)$ in the sequence (44). down to approximately zero energy at a sequence of "magic" twist angles. ${ }^{44,45}$ The first instance at which this happens corresponds to $n=31$. Then, in the way towards this magic angle, the lowest subband of the bilayer becomes increasingly flat, which has a significant impact on the values of the electron-hole susceptibility. This can be appreciated in the plot of Fig. 10(a), which shows the result of computing $\chi_{\mathrm{ph}}(\mathbf{q})$ for a twisted bilayer with $n=22$. From the position of the pole in the spin response function $R_{S}(\mathbf{0}, \omega)$, one can calculate again the critical values of the interaction strength needed to reach the magnetic instability. These are represented in Fig. 10(b) as a function of the temperature and the deviation of the filling level with respect to the Van Hove singularity. With a rough estimate of $U / M \sim 0.007 \mathrm{eV}$ for the corresponding twisted bilayer, we find that the onset of the instability may now take place at temperatures approaching the order of magnitude of $10 \mathrm{~K}$.

It is interesting to observe that, from the point of view of the real space, the limit of very small twist angle studied here is characterized by a well-defined pattern of localization in the moire superlattice, ${ }^{45,56,57}$ such that the states in the lowest-energy subband tend to be confined to the regions with regular $A A$ stacking (where all the atoms in one layer are in registry with their homologous in the other layer). This localization of the wave functions is the counterpart of the progressive narrowing of the lowest subband, until it becomes flat at the first magic angle. The particular pattern of confinement is actually consistent with the tendency towards ferromagnetism driven by the Van Hove singularity, as the regions with regular $A A$ stacking form a triangular superlattice that is incompatible with a staggered order of the spins (implied for instance by a antiferromagnetic instability). Indeed, the localization of the wave functions, together with the quench in kinetic energy due to the formation of the narrow subband, is an effect that favors the uniform alignment of the spins. In a Hubbard-like description of this correlated behavior in the bilayer superlattice, the effective Coulomb interaction has to scale in inverse proportion to the number of carbon atoms covered by the confined states in a unit cell, as the probability of the electron occupancy for a single atom is reduced by that number. This is consistent with the above estimate of the effective Coulomb repulsion, which justifies our computational approach when such interaction strength is much smaller than the bandwidth of the electron system. This certainly happens in the two cases considered with $n=10$ and 22. As the bandwidth goes to zero, the scaling approach must break down however at some larger lattice constant of the twisted bilayer, leaving then ferromagnetism as a likely instability but much harder to analyze quantitatively.

\section{CONCLUSIONS}

In this paper we have studied the many-body instabilities of electrons interacting near Van Hove singularities arising in monolayer and twisted bilayer graphene. In the first instance, we have taken advantage of the experimental data available for the dispersion around the saddle points in the conduction band of graphene. ${ }^{29}$ While reaching there the level of the singularity requires a large amount of doping, the results obtained from ARPES have unveiled the extended character of the saddle-point dispersion, showing the potential for a large 
instability of the electron system. On the other hand, twisted graphene bilayers have Van Hove singularities that arise from the hybridization of the Dirac cones of the two layers, being therefore located at relatively low energies from the charge neutrality point. They can be more easily reached upon doping, but have the disadvantage of involving in general a weaker singularity in the density of states, with lower strength of the consequent electronic instabilities.

We have seen that a pairing instability must be dominant over the tendency to magnetic order as the Fermi level is tuned to the Van Hove singularity in the conduction band of graphene. As a result of the extended character of the saddle points in the dispersion, we have found that the pairing of the electrons must take place preferentially in a channel of $f$-wave symmetry, with an order parameter vanishing at the position of the saddle points along the Fermi line. In the case of the twisted bilayers, the dispersion has instead its symmetry reduced down to the $C_{3 v}$ group and, most importantly, it leads to susceptibilities that diverge at the saddle points but are integrable along the Fermi line. This implies that the attractive couplings for the pairing instability do not grow large when approaching the critical interaction strength marking the onset of magnetic order. Thus, the magnetic instability becomes prevalent in the twisted graphene bilayers, with a dominant tendency towards ferromagnetism as the uniform magnetic susceptibility inherits the divergence in the density of states at the Van Hove singularity.

The divergence of the particle-hole and particle-particle susceptibilities is the driving force for magnetic and pairing instabilities at the Van Hove singularity. In order to account for the latter, we have relied on the sum of the most divergent contributions to the BCS vertex in the particle-particle channel, which arise from the iteration of the scattering of the pair of electrons. Assuming a purely repulsive interaction, the series of corrections built in that way does not give rise in principle to any singularity in the vertex, but the approach can be significantly improved by considering that the scattering is mediated by the effective RPA screened interaction. This leads to a much more comprehensive approximation, in which the $f$-wave pairing instability we have obtained can be interpreted as being induced by the ferromagnetic fluctuations which grow increasingly large near the singularity of the RPA series.

Conversely, one may also ask about the possible effect of higher order corrections to the RPA description of the ferromagnetic instability. These can play an important role in the present context, since particle-particle loops are enhanced as the square of the logarithm of the energy near the Van Hove singularity. While the general analysis of their effect becomes practically unfeasible, one can identify that an important set of contributions corresponds to electron self-energy corrections. These are enhanced by $\log$-square terms to second order in perturbation theory, with the potential to suppress the electron quasiparticle weight at low energies. ${ }^{48}$ However, there is a concomitant effect arising from the renormalization of the saddle-point dispersion, which tends to become even flatter at low energies, amplifying therefore the ferromagnetic fluctuations. We may conclude that while the RPA is equivalent to a lowest-order renormalization group approach and therefore reliable for a Coulomb repulsion much lower than the energy scale of the saddle-point dispersion, the low-energy behavior of the system becomes more unpredictable away from such weak-coupling regime. More elaborated analyses carried out in the square lattice, relying on functional renormalization ${ }^{58}$ or on nonperturbative treatments based on Monte Carlo simulations, ${ }^{46}$ have asserted anyhow the existence of the ferromagnetic instability in the case of a dominant particlehole susceptibility at vanishing momentum transfer near the Van Hove singularity.

In practice, there is also a restriction in the growth of the different susceptibilities coming from the effect of disorder or defects in the carbon lattice. These have the ability to smear the singularity in the density of states, reducing its nominal strength. The most dangerous effect comes in that respect from the scattering off impurities, and the analysis made in the two-dimensional square lattice has shown that the logarithmic singularity in the density of states is suppressed by corrections that depend on the function $(1 / 4 \pi t \tau) \ln (4 \tau|\varepsilon|)$, $t$ being the nearest-neighbor hopping amplitude and $\tau$ the relaxation time. ${ }^{59}$ In our case, for sufficiently clean samples, we can assume for instance a value of the mean-free path in the carbon lattice of the order of $\sim 1 \mu \mathrm{m}$, which would imply a relaxation time $\tau \sim 10^{4} t^{-1}$. Thus, we see that the divergence in the density of states may not suffer a significant attenuation when probed with a resolution of $\sim 0.1 \mathrm{meV}$. This level of approximation to the singularity may be small enough to observe the onset of the electronic instabilities since, as shown above, it corresponds to doping levels at which experimental signatures can be seen for reasonable values of the interaction strength in monolayer as well as in twisted bilayer graphene.

The results presented in this paper may serve as a guide for the effects that can be measured in real graphene samples near a Van Hove singularity. Renormalization group methods have been shown to provide in general a valuable approach to the low-energy physics of the singular density of states, where effects like the quasiparticle attenuation may be compensated in part by the renormalization of the saddle-point dispersion. ${ }^{48}$ The carbon-based systems studied in the present paper may provide an appropriate playground to test many of the physical effects predicted, including the regime of strong correlations supposed to arise in the vicinity of the Van Hove singularity.

\section{ACKNOWLEDGMENTS}

I thank P. San-José for his support at the early stages of the work on twisted graphene bilayers. I also thank F. Guinea for very useful conversations. The financial support from MICINN (Spain) through Grant No. FIS2011-23713 is gratefully acknowledged.
${ }^{1}$ D. V. Khveshchenko, Phys. Rev. Lett. 87, 246802 (2001).

${ }^{2}$ I. F. Herbut, V. Juričić, and O. Vafek, Phys. Rev. B 80, 075432 (2009).
${ }^{3}$ V. Juričić, I. F. Herbut, and G. W. Semenoff, Phys. Rev. B 80, 081405 (2009).

${ }^{4}$ J. E. Drut and T. A. Lähde, Phys. Rev. Lett. 102, 026802 (2009). 
${ }^{5}$ S. J. Hands and C. G. Strouthos, Phys. Rev. B 78, 165423 (2008).

${ }^{6}$ O. V. Gamayun, E. V. Gorbar, and V. P. Gusynin, Phys. Rev. B 80, 165429 (2009).

${ }^{7}$ J. Wang, H. A. Fertig, and G. Murthy, Phys. Rev. Lett. 104, 186401 (2010).

${ }^{8}$ O. V. Gamayun, E. V. Gorbar, and V. P. Gusynin, Phys. Rev. B 81, 075429 (2010).

${ }^{9}$ J. González, Phys. Rev. B 85, 085420 (2012).

${ }^{10}$ O. Vafek and K. Yang, Phys. Rev. B 81, 041401(R) (2010).

${ }^{11}$ F. Zhang, H. Min, M. Polini, and A. H. MacDonald, Phys. Rev. B 81, 041402(R) (2010).

${ }^{12}$ R. Nandkishore and L. Levitov, Phys. Rev. Lett. 104, 156803 (2010).

${ }^{13}$ Y. Lemonik, I. L. Aleiner, C. Toke, and V. I. Fal'ko, Phys. Rev. B 82, 201408(R) (2010).

${ }^{14}$ V. N. Kotov, B. Uchoa, V. M. Pereira, F. Guinea, and A. H. Castro Neto, Rev. Mod. Phys. 84, 1067 (2012).

${ }^{15}$ X. Du, I. Skachko, F. Duerr, A. Luican, and E. Y. Andrei, Nature (London) 462, 192 (2009).

${ }^{16}$ K. I. Bolotin, F. Ghahari, M. D. Shulman, H. L. Stormer, and P. Kim, Nature (London) 462, 196 (2009).

${ }^{17}$ A. S. Mayorov, D. C. Elias, M. Mucha-Kruczynski, R. V. Gorbachev, T. Tudorovskiy, A. Zhukov, S. V. Morozov, M. I. Katsnelson, V. I. Fal'ko, A. K. Geim, and K. S. Novoselov, Science 333, 860 (2011).

${ }^{18}$ J. Velasco, Jr., L. Jing, W. Bao, Y. Lee, P. Kratz, V. Aji, M. Bockrath, C. N. Lau, C. Varma, R. Stillwell, D. Smirnov, F. Zhang, J. Jung, and A. H. MacDonald, Nat. Nanotechnol. 7, 156 (2012).

${ }^{19}$ J. González, F. Guinea, and M. A. H. Vozmediano, Phys. Rev. B 63, 134421 (2001).

${ }^{20}$ B. Uchoa and A. H. Castro Neto, Phys. Rev. Lett. 98, 146801 (2007).

${ }^{21}$ A. M. Black-Schaffer and S. Doniach, Phys. Rev. B 75, 134512 (2007).

${ }^{22}$ C. Honerkamp, Phys. Rev. Lett. 100, 146404 (2008).

${ }^{23}$ J. González, Phys. Rev. B 78, 205431 (2008).

${ }^{24}$ B. Roy and I. F. Herbut, Phys. Rev. B 82, 035429 (2010).

${ }^{25}$ F. Guinea and B. Uchoa, Phys. Rev. B 86, 134521 (2012).

${ }^{26}$ B. Uchoa and Y. Barlas, Phys. Rev. Lett. 111, 046604 (2013).

${ }^{27}$ H. B. Heersche, P. Jarillo-Herrero, J. B. Oostinga, L. M. K. Vandersypen, and A. F. Morpurgo, Nature (London) 446, 56 (2007).

${ }^{28}$ A. Shailos, W. Nativel, A. Kasumov, C. Collet, M. Ferrier, S. Guéron, R. Deblock, and H. Bouchiat, Europhys. Lett. 79, 57008 (2007).

${ }^{29}$ J. L. McChesney, A. Bostwick, T. Ohta, T. Seyller, K. Horn, J. González, and E. Rotenberg, Phys. Rev. Lett. 104, 136803 (2010).

${ }^{30}$ G. Li, A. Luican, J. M. B. Lopes dos Santos, A. H. Castro Neto, A. Reina, J. Kong, and E. Y. Andrei, Nat. Phys. 6, 109 (2010).

${ }^{31}$ W. Yan, W.-Y. He, Z.-D. Chu, M. Liu, L. Meng, R.-F. Dou, Y. Zhang, Z. Liu, J.-C. Nie, and L. He, Nat. Commun. 4, 2159 (2013).

${ }^{32}$ W. Kohn and J. M. Luttinger, Phys. Rev. Lett. 15, 524 (1965).
${ }^{33}$ M. A. Baranov, A. V. Chubukov, and M. Yu. Kagan, Int. J. Mod. Phys. B 6, 2471 (1992).

${ }^{34}$ J. González, F. Guinea, and M. A. H. Vozmediano, Phys. Rev. Lett. 79, 3514 (1997).

${ }^{35}$ K. Gofron, J. C. Campuzano, A. A. Abrikosov, M. Lindroos, A. Bansil, H. Ding, D. Koelling, and B. Dabrowski, Phys. Rev. Lett. 73, 3302 (1994).

${ }^{36}$ Z.-X. Shen, W. E. Spicer, D. M. King, D. S. Dessau, and B. O. Wells, Science 267, 343 (1995).

${ }^{37}$ D. M. Newns, H. R. Krishnamurthy, P. C. Pattnaik, C. C. Tsuei, and C. L. Kane, Phys. Rev. Lett. 69, 1264 (1992).

${ }^{38}$ R. S. Markiewicz, J. Phys. Chem. Solids 58, 1179 (1997).

${ }^{39}$ J. González, F. Guinea, and M. A. H. Vozmediano, Europhys. Lett. 34, 711 (1996).

${ }^{40}$ D. Zanchi and H. J. Schulz, Phys. Rev. B 54, 9509 (1996).

${ }^{41}$ R. Nandkishore, L. Levitov, and A. Chubukov, Nat. Phys. 8, 158 (2012).

${ }^{42}$ M. L. Kiesel, C. Platt, W. Hanke, D. A. Abanin, and R. Thomale, Phys. Rev. B 86, 020507 (2012).

${ }^{43}$ J. M. B. Lopes dos Santos, N. M. R. Peres, and A. H. Castro Neto, Phys. Rev. Lett. 99, 256802 (2007).

${ }^{44}$ R. Bistritzer and A. H. MacDonald, Proc. Natl. Acad. Sci. USA 108, 12233 (2011).

${ }^{45}$ P. San-José, J. González, and F. Guinea, Phys. Rev. Lett. 108, 216802 (2012).

${ }^{46}$ R. Hlubina, S. Sorella, and F. Guinea, Phys. Rev. Lett. 78, 1343 (1997).

${ }^{47}$ J. V. Alvarez, J. González, F. Guinea, and M. A. H. Vozmediano, J. Phys. Soc. Jpn 67, 1868 (1998).

${ }^{48}$ J. González, F. Guinea, and M. A. H. Vozmediano, Nucl. Phys. B 485, 694 (1997).

${ }^{49}$ D. J. Scalapino, E. Loh, and J. E. Hirsch, Phys. Rev. B 35, 6694 (1987).

${ }^{50}$ R. Hlubina, Phys. Rev. B 59, 9600 (1999).

${ }^{51}$ J. Hass, F. Varchon, J. E. Millán-Otoya, M. Sprinkle, N. Sharma, W. A. de Heer, C. Berger, P. N. First, L. Magaud, and E. H. Conrad, Phys. Rev. Lett. 100, 125504 (2008).

${ }^{52}$ A. Luican, G. Li, A. Reina, J. Kong, R. R. Nair, K. S. Novoselov, A. K. Geim, and E. Y. Andrei, Phys. Rev. Lett. 106, 126802 (2011).

${ }^{53}$ E. J. Mele, Phys. Rev. B 81, 161405(R) (2010).

${ }^{54}$ S. Shallcross, S. Sharma, E. Kandelaki, and O. A. Pankratov, Phys. Rev. B 81, 165105 (2010).

${ }^{55}$ T. O. Wehling, E. Şaşioğlu, C. Friedrich, A. I. Lichtenstein, M. I. Katsnelson, and S. Blügel, Phys. Rev. Lett. 106, 236805 (2011).

${ }^{56}$ G. Trambly de Laissardière, D. Mayou, and L. Magaud, Nano Lett. 10, 804 (2010)

${ }^{57}$ S. Shallcross, S. Sharma, and O. Pankratov, Phys. Rev. B 87, 245403 (2013).

${ }^{58}$ C. Honerkamp and M. Salmhofer, Phys. Rev. B 64, 184516 (2001).

${ }^{59}$ E. P. Nakhmedov, M. Kumru, and R. Oppermann, Phys. Rev. Lett. 84, 3930 (2000). 